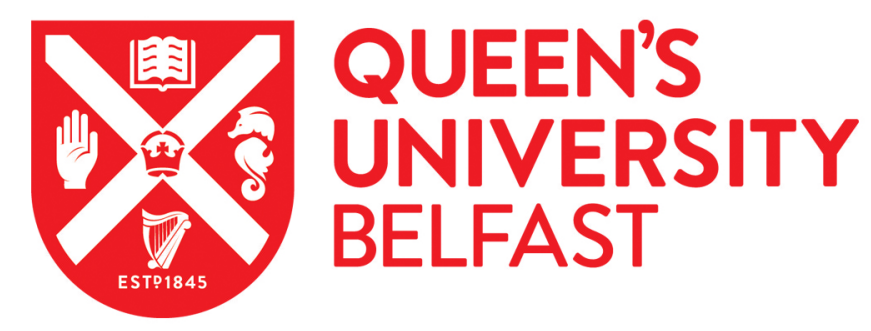

\title{
Optical Contrast of Atomically Thin Films
}

Huang, F. (2019). Optical Contrast of Atomically Thin Films. The Journal of Physical Chemistry C. https://doi.org/10.1021/acs.jpcc.8b12333

\section{Published in:}

The Journal of Physical Chemistry C

\section{Document Version:}

Peer reviewed version

Queen's University Belfast - Research Portal:

Link to publication record in Queen's University Belfast Research Portal

\section{Publisher rights}

(c) 2019 American Chemical Society. This work is made available online in accordance with the publisher's policies. Please refer to any applicable terms of use of the publisher.

\section{General rights}

Copyright for the publications made accessible via the Queen's University Belfast Research Portal is retained by the author(s) and / or other copyright owners and it is a condition of accessing these publications that users recognise and abide by the legal requirements associated with these rights.

Take down policy

The Research Portal is Queen's institutional repository that provides access to Queen's research output. Every effort has been made to ensure that content in the Research Portal does not infringe any person's rights, or applicable UK laws. If you discover content in the Research Portal that you believe breaches copyright or violates any law, please contact openaccess@qub.ac.uk. 


\title{
THE JOURNAL OF PHYSICAL CHEMISTRY
}

Subscriber access provided by QUEENS UNIV BELFAST

\section{C: Physical Processes in Nanomaterials and Nanostructures}

\section{Optical Contrast of Atomically Thin Films}

\author{
Fumin Huang
}

J. Phys. Chem. C, Just Accepted Manuscript • DOI: 10.1021/acs.jpcc.8b12333 • Publication Date (Web): 07 Mar 2019

Downloaded from http://pubs.acs.org on March 12, 2019

\section{Just Accepted}

"Just Accepted" manuscripts have been peer-reviewed and accepted for publication. They are posted online prior to technical editing, formatting for publication and author proofing. The American Chemical Society provides "Just Accepted" as a service to the research community to expedite the dissemination of scientific material as soon as possible after acceptance. "Just Accepted" manuscripts appear in full in PDF format accompanied by an HTML abstract. "Just Accepted" manuscripts have been fully peer reviewed, but should not be considered the official version of record. They are citable by the Digital Object Identifier (DOI®). "Just Accepted" is an optional service offered to authors. Therefore, the "Just Accepted" Web site may not include all articles that will be published in the journal. After a manuscript is technically edited and formatted, it will be removed from the "Just Accepted" Web site and published as an ASAP article. Note that technical editing may introduce minor changes to the manuscript text and/or graphics which could affect content, and all legal disclaimers and ethical guidelines that apply to the journal pertain. ACS cannot be held responsible for errors or consequences arising from the use of information contained in these "Just Accepted" manuscripts. 


\title{
Optical Contrast of Atomically Thin Films
}

\author{
Fumin Huang* \\ School of Mathematics and Physics, Queen's University Belfast, Belfast, United Kingdom, BT7 \\ $1 \mathrm{NN}$
}

\begin{abstract}
Here we provide a comprehensive description of the optical contrast of atomicallythin films, based on rigorous analytical solutions. The effects of thin film, substrate and light illumination conditions are fully revealed. The role of substrate is found to be completely represented by a single complex reflectivity, regardless of the structural details. High contrast is realized on low-reflection substrates; however, the phase of the complex reflectivity is critically important. Every thin film has specific reflectivity-phase conditions to achieve optimal contrast, which are uniquely defined by the optical properties of the thin film. Extraordinarily high optical contrast can be achieved on any thin film of any thickness, if the reflectivity of substrates matches the optimal phase conditions. We provide a universal phase map which can be used to determine the optimal phases of any given film. For example, the optimal phase of hexagonal boron nitride $(\mathrm{hBN})$ films is found to be $-90^{\circ}$, which paves the way towards designing high-contrast substrates to visualize this highly transparent 2D material. We also provide detailed discussions on the effects of a range of other factors, including polarizations, incident angles, and the numerical aperture of objectives.
\end{abstract}




\section{INTRODUCTION}

Visualizing and accurately determining the thickness of atomically-thin films is central in research and applications of two-dimensional (2D) materials, such as graphene, ${ }^{1-3}$ hexagonal boron nitride $(\mathrm{hBN}){ }^{4}$ black phosphorous $(\mathrm{BP}){ }^{5}$ and a wide variety of transition metal dichalcogenides (TMDs). ${ }^{6,7}$ Many bulk 2D materials have layer-by-layer crystal structures bonded by weak van der Waals forces, hence monolayers or few-layer films can be exfoliated with mechanical or chemical methods, ${ }^{7-9}$ producing extremely thin $2 \mathrm{D}$ crystals with thickness between one atom and a few nanometers. These $2 \mathrm{D}$ films possess extraordinary properties not attainable in their bulk counterparts. One prominent example is graphene, a single-atom graphite layer, which however has better electrical and thermal conductivities than silver and is mechanically stronger than steel. ${ }^{10}$ Another example is TMDs. They are often indirect-bandgap semiconductors as bulk materials, but transferring to direct-bandgap semiconductors in monolayer forms, making them wonderful candidates for photonic devices. ${ }^{11}$ Till date, several hundreds of species of 2D materials have been discovered. ${ }^{11-13}$ The vast variety of $2 \mathrm{D}$ materials provide a plethora of fascinating properties, making promising materials for revolutionary applications in many industrial sectors, such as optoelectronics, sensors and energy. ${ }^{10-13}$ As the functionalities of $2 \mathrm{D}$ films are critically dependent on the number of layers, developing facile technologies to visualize and to rapidly and accurately determine the thickness of atomically-thin films is instrumentally important for the field of $2 \mathrm{D}$ materials. 
Conventionally the thickness of thin films can be characterized with a variety of scanning probe microscopies, such as atomic force microscopy (AFM), scanning electron microscopy (SEM) and transmission electron microscopy (TEM), but these methods are time-consuming and costly, requiring expensive equipment. In comparison, optical contrast spectroscopy (OCS) is a facile technique with many advantages, which can characterize film thickness non-invasively with minimal time, little cost and high accuracy. As such, it has become one of the most important thinfilm characterization tools widely adopted in the determination of 2D materials thickness. ${ }^{1-6}$

Despite much research has been carried out on OCS, a comprehensive description of the technique from fundamental optic principles has not been provided and there are a number of outstanding issues to be solved. For example, many measurements were taken with objectives of large numerical apertures (NA) spanning a broad range of incident angles, however quite often Fresnel reflection formulae at normal incidence were used to model the results, leading to notable discrepancy between theory and experiments. ${ }^{1,14}$ A second imminent issue is, although OCS has been successfully demonstrated on a number of materials, ${ }^{1-6}$ it is still challenging on some extremely transparent materials, such as hBN. Till date, $\mathrm{hBN}$ is one of most difficult materials to visualize, with recorded maximum contrast only about $2.5 \%$ for a monolayer film. ${ }^{4}$ Most optical characterizations of hBN have been limited to multilayers. ${ }^{15,16}$ Finding the optimal conditions to achieve high contrast of $\mathrm{hBN}$ is central to the further development of this intriguing nanomaterial. ${ }^{17,18}$ As will be shown in this paper, even for films that are highly visible on some substrates, they could be invisible on different substrates. For instance, graphene is readily visible on $\mathrm{SiO}_{2}$-coated $\mathrm{Si}^{1,14}$ but hard to see on highly reflective metal surface. ${ }^{2,3}$ The rapid advance of 
the field of 2D materials has empowered research and applications on a broad variety of materials, as the functionalities of 2D material devices are critically dependent on the underlying substrate material which the 2D materials are directly in contact, which could impact the electronic, optical, catalytic and electrochemical properties of 2D films through doping, strain and energy transfer processes. ${ }^{19,20}$ Currently contrast-enhancing substrates are mainly limited to oxidized Si and a few metallic structures. It is therefore desirable to develop high-contrast substrates with wide options of top-surface materials. To accomplish all these, a deep understanding of the fundamentals of the optical contrast of thin films is essential.

Here we provide a comprehensive description of the optical contrast of ultrathin films. The effects of thin film, substrate, and light illumination conditions (wavelength, incident angle, and polarization) are fully investigated. It is shown, the role of substrates is completely represented by a single complex reflectivity, regardless of the structural details of the substrate, i.e., two substrates of different structures will produce same optical contrast for a given thin film, if they produce same complex reflectivity. In general, contrast increases with decreasing reflectivity of substrates, therefore optimal contrast will be achieved on substrates of minimum reflection. However, the phase of substrate reflectivity is crucially important. For any thin film, optimal contrast is only achievable at specific reflectivity phases uniquely decided by the optical properties of the thin film. Apart from substrates and thin films, optical contrast also depends on the illumination conditions of light, including wavelength, incident angles, and polarizations. To correctly interpret experimental results, all these effects need to be properly taken into account. The results in this paper demonstrate that, with appropriately designed substrates, extremely high optical contrast can be achieved on any film of any thickness. 


\section{THEORY}

Optical contrast is induced by the reflectance difference of light on a substrate with and without a thin film (Fig.1), which is defined as

$$
C=1-\frac{R^{\prime}}{R}
$$

$R^{\prime}=\left|r^{\prime}\right|^{2}$ and $R=|r|^{2}$, with $r^{\prime}$ and $r$ being the complex reflectivity with and without the thin film, respectively. A positive contrast is achieved for $R^{\prime}<R$, which means less light is reflected on the area covered with thin films in comparison to the bare substrate. Conversely a negative contrast is achieved for $R^{\prime}>R$, corresponding to more light is reflected on the area covered with thin films. With this definition, the upper limit of positive contrast is unity (when $R^{\prime}=0$ ), whereas the negative contrast is unbounded, up to $-\infty$ (when $R=0$ ).

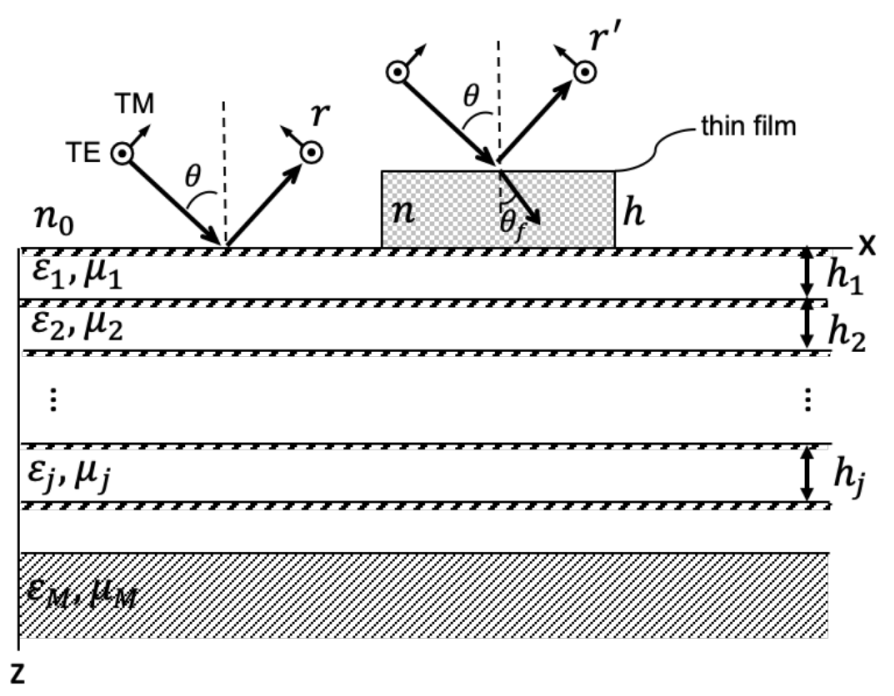

Figure 1 Schematic of light reflection on a stratified substrate with and without a thin film. The substrate is composed of $M$ layers of homogeneous planar films with optical properties denoted by $\varepsilon_{j}, u_{j}$, and thickness by $h_{j}, j$ is the layer number. 
It is convenient to introduce a quantity denoting the relative change in reflectivity, $\chi=1-\frac{r^{\prime}}{r} \equiv$ $\chi_{0} e^{i \psi}$. The contrast then is obtained as

$$
C=2 \chi_{0} \cos \psi-\chi_{0}^{2}
$$

which is related to both the magnitude and phase of $\chi$.

For a stratified substrate composed of multilayer homogeneous planar films (the multilayer structure allows to generate desired complex reflectivity $r$ by tuning the optical properties and thickness of the constituent layers ${ }^{3}$ ) as schematically depicted in Fig.1, $r^{\prime}$ is uniquely determined by $r$ and the thin film. $\chi$ can be analytically expressed as (see Supplementary Information for derivation),

$$
\chi=\frac{\frac{1+r}{r}\left[\eta^{2}-\left(\frac{1-r}{1+r}\right)^{2}\right] \tanh (-i \beta)}{\frac{2 \eta}{1+r}+\left[\frac{1-r}{1+r}+\eta^{2}\right] \tanh (-i \beta)}
$$

$\beta=n k_{0} h \cos \theta_{f} \cdot n, h, \theta_{f}$ are the refractive index (RI), thickness and refraction angle inside the thin film, as depicted in Fig.1. $k_{0}=\frac{2 \pi}{\lambda}, \lambda$ is the wavelength of light in free space. For TMpolarization $\eta=\frac{n_{0} \cos \theta_{f}}{n \cos \theta} \equiv \xi$ and for TE-polarization $\eta=\frac{n \cos \theta_{f}}{n_{0} \cos \theta} \equiv \zeta, n_{0}$ is the RI of the surrounding medium and $\theta$ the incident angle. In the following, we focus discussions mainly on TE-polarization. The results of TM-polarization can be obtained straightforwardly by replacing $\zeta$ with $\xi$. The above formulae are exact without any approximation, which are applicable to any homogeneous thin film, any stratified substrate and arbitrary light illumination conditions. 
In the case of ultrathin films like $2 \mathrm{D}$ materials, $k_{0} h \ll 1$, so that $\beta \ll 1, \tanh (-i \beta) \approx-i \beta$. The second term in the denomenator of Eq.3 is negilible comparing to the first term, so Eq.3 can be simplified as

$$
\chi \approx-i \beta \frac{\frac{1+r}{r}\left[\eta^{2}-\left(\frac{1-r}{1+r}\right)^{2}\right]}{\frac{2 \eta}{1+r}}
$$

By multiplying $r(1+r)$ on both the numerator and denomenator of Eq.4a, we obtain

$$
\chi \approx-i \beta \frac{(1+r)^{2} \eta^{2}-(1-r)^{2}}{2 \eta r}
$$

For TE-polarization, replacing $\eta$ with $\zeta=\frac{n \cos \theta_{f}}{n_{0} \cos \theta}$ and applying the Snell's law of reflection, we have

$$
\begin{gathered}
\chi=-i \frac{k_{0} h}{2 r \cos \theta} \delta \\
\delta=(1+r)^{2}\left(n^{2}-\sin ^{2} \theta\right)-\cos ^{2} \theta(1-r)^{2}
\end{gathered}
$$

Let $\delta \equiv \delta_{0} e^{i \phi}, r \equiv r_{0} e^{i \varphi}$, from Eq.5a it is inferred

$$
\begin{aligned}
& \chi_{0}=\frac{k_{0} h \delta_{0}}{2 r_{0} \cos \theta} \\
& \psi=\phi-\varphi-\frac{\pi}{2} \pm 2 m \pi
\end{aligned}
$$

$m$ is an integer so that $0 \leq \psi<2 \pi$. For simplicity, we assume $n_{0}=1$ in obtaining the above results. If the surrounding medium is not air, $n$ should be considered as a reduced RI normalized to surrounding medium. 
The above formulae apply excellently on few-layer 2D materials, as the condition $k_{0} h \ll 1$ is well matched, e.g., let $h=1 \mathrm{~nm}$ and $\lambda=500 \mathrm{~nm}$, then $k_{0} h \approx 0.012$.

\section{RESULTS}

\section{Effects of substrate}

As seen from Eq.6a, $\chi_{0}$ is inversely proportional to $r_{0}$, so in general a low-reflection substrate will provide a high contrast. However, the phase of $\chi$ (hence the phase $\varphi$ of $r$, according to Eq.6b) is also important, as indicated in Eq.2. The effect of $\varphi$ can be explicitly illustrated by keeping $\chi_{0}$ constant. Based on Eq.2, maximum positive (negative) contrast will be achieved at $\psi=0(\pi)$. This leads to the optimal phases $\varphi^{ \pm}$and optimal contrasts $C^{ \pm}$as following

$$
\begin{aligned}
& \varphi^{ \pm}=\phi \mp \frac{\pi}{2} \pm 2 m \pi \\
& C^{ \pm}= \pm 2 \chi_{0}-\chi_{0}^{2}
\end{aligned}
$$

$\varphi^{+}$and $\varphi^{-}$differ by a factor of $\pi$, which are decided only by $\phi$ (the phase of $\delta$ ). In the case of $r_{0} \ll 1$ (a necessary condition for high-contrast substrates), the $r$ terms in Eq.5b are negilible, therefore

$$
\delta \approx n^{2}-1
$$

Eq.8 indicates that $\phi$ is solely determined by $n$ and independent on the incident angle. Eqs. $(7,8)$ suggest, every thin film has specific optimal phases $\left(\varphi^{ \pm}\right)$for high contrast, which are uniquely determined by the optical properties $(n)$ of the thin film. Ideally a high-contrast substrate should have a low reflectivity with the optimal phases given in Eq.7a. This is universal for any thin film. 

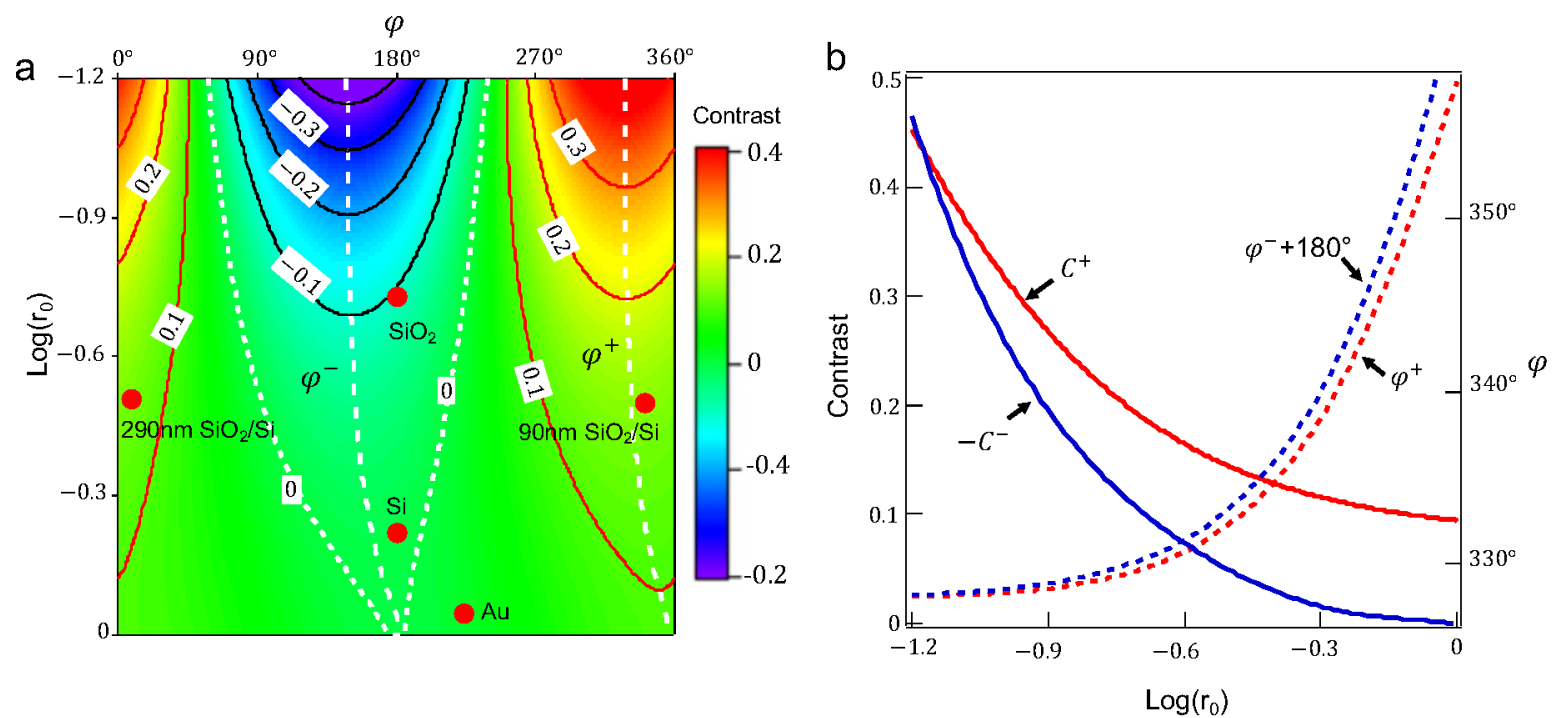

Figure 2 Contrast map of graphene. (a) Calculated contrast of monolayer graphene on substrates of complex reflectivity $r=r_{0} e^{i \varphi}$. Numbers on contour plots indicate the contrast. Traces of optimal phases $\varphi^{ \pm}$are indicated with dashed lines. The dotted line indicates the track of zero contrast. A few commonly-used substrates are marked by symbols. $\lambda=560 \mathrm{~nm}, \theta=0^{\circ}$, TE-polarization. Thickness of graphene is $h=0.34 \mathrm{~nm}$ and $n=2.6+1.3 i$. (b) The maximum contrast $C^{ \pm}$and the corresponding phase $\varphi^{ \pm}$along the dashed lines in $\mathbf{a}$.

As the effect of substrate is completely represented by a single complex reflectivity $r$, the contrast of a thin film can be fully evaluated based on $r$. Figure 2 shows the calculated contrast map (normal incidence, $\lambda=560 \mathrm{~nm}$ ) of a monolayer graphene with regard to the magnitude and phase of the complex reflectivity $r$ of substrates. Generally contrast increases with decreasing $r_{0}$, consistent with the discussions above. However, the phase of $r$ is also critically important. As seen from Fig.2a, no matter how small $r_{0}$ is, there always exist phases at which the contrast is zero (dotted lines). Optimal contrasts are localized in two distinct phase regions (blue and red areas). The maximum positive/negative contrasts are marked by dashed lines and plotted in Fig. 
$2 \mathrm{~b}$, both increasing with diminished reflectivity $r_{0}$. For substrates of small reflectivity $\left(r_{0} \ll 1\right)$, optimal phases are nearly constant, e.g., for graphene, $\varphi^{+} \approx 328^{\circ}$ and $\varphi^{-} \approx 148^{\circ}$.

With the contrast map, the visibility of graphene on any substrate can be assessed. The contrasts of a few commonly-used substrates are highlighted on the map of Fig.2a, including $90 \mathrm{~nm}$ $\mathrm{SiO}_{2} / \mathrm{Si}, 290 \mathrm{~nm} \mathrm{SiO} / / \mathrm{Si}, \mathrm{SiO}_{2}, \mathrm{Si}$ and $\mathrm{Au}$. Oxidized $\mathrm{Si}$ substrates provide $~ 14 \%$ contrast for a monolayer graphene, while Si and Au only provide a few percentages, which are consistent with experimental results. ${ }^{1-3}$ This indicates that contrast critically relies on substrates. A film highly visible on one substrate could be invisible on another. Conversely, a film extremely transparent on conventional substrates can be made highly visible on appropriately-designed contrastboosting substrates.

\section{Optimal phases}

According to Eq.8, the optimal phases of a thin film are solely decided by the complex refractive index of the thin film, $n=n_{1}+i n_{2}$. Figure 3 shows the map of $\varphi^{+}\left(\varphi^{-}\right.$is $180^{\circ}$ difference $)$ with regard to $n$. It is seen that $\varphi^{+}$shifts clockwise with increasing $n_{2}$ when $n_{1}$ is fixed and anticlockwise with increasing $n_{1}$ when $n_{2}$ is fixed. All the contour lines converge to a singular point at $n=1$ (a virtual film of free space), where the phase is undefined as the contrast is zero. With this universal map, the optimal phases of any given thin film can be determined if the refractive index is known. To give examples, the optimal phases of a number of 2D materials are highlighted on the map, based on the refractive indices listed in Table 1. 


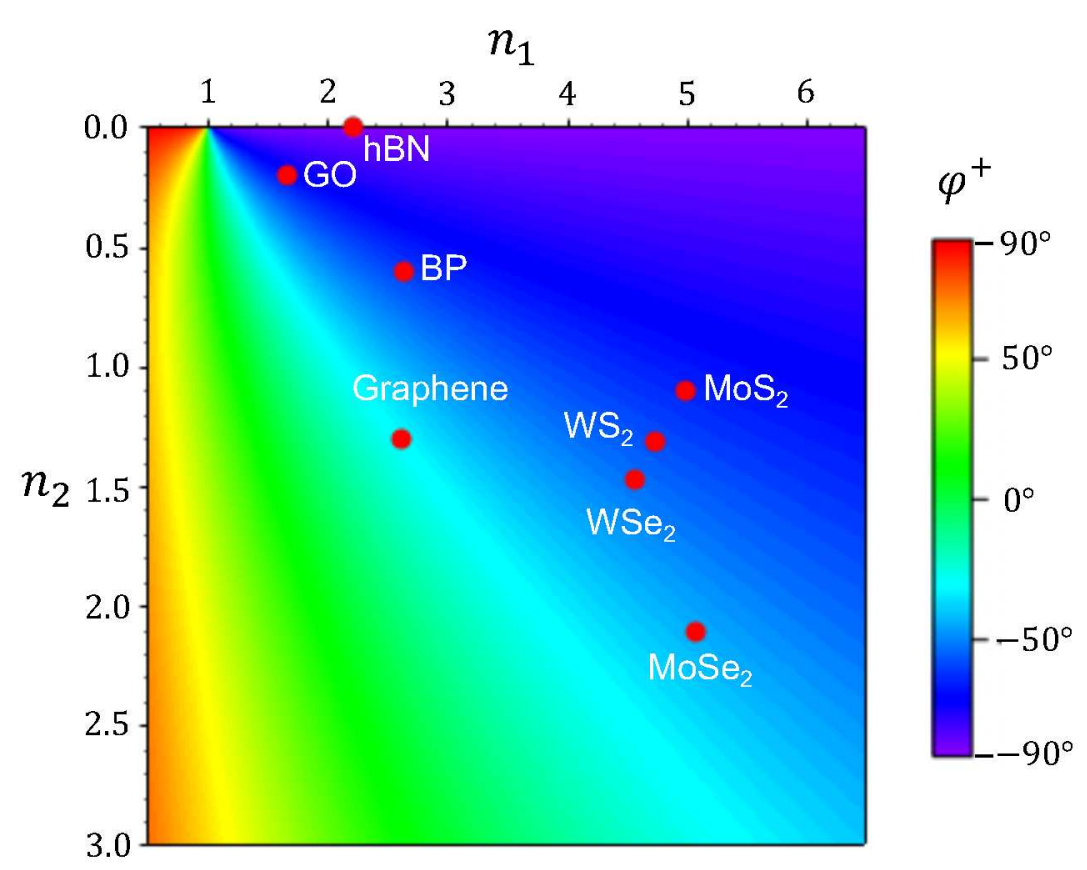

Figure 3 Map of optimal phases. Calculated optimal phase $\left(\varphi^{+}\right)$with regard to the complex refractive index of thin film $n=n_{1}+i n_{2}$. The optimal phases of a number of $2 \mathrm{D}$ materials are highlighted. The refractive indice of $2 \mathrm{D}$ materials are shown in Table 1.

Table 1 Optimal phases of 2D materials. Refractive indices are adopted from the annotated refs. For TMDs, all $n$ is for $\lambda=520 \mathrm{~nm}$.

\begin{tabular}{|c|c|c|}
\hline 2D materials & $n$ & $\varphi^{+}\left(^{\circ}\right)$ \\
\hline Graphene (Gr) & $2.6+1.3 i^{1}$ & -31 \\
\hline Graphene oxide (GO) & $1.65+0.2 i^{3}$ & -67 \\
\hline hBN & $2.2^{14}$ & -90 \\
\hline Black phosphrous (BP) & $2.64+0.6 i^{21}$ & -60 \\
\hline MoS $_{2}$ & $4.98+1.1 i^{22}$ & -64 \\
\hline $\mathrm{WS}_{2}$ & $4.74+1.31 i^{22}$ & -57 \\
\hline $\mathrm{WSe}_{2}$ & $4.57+1.47 i^{22}$ & -53 \\
\hline $\mathrm{MoSe}_{2}$ & $5.08+2.1 i^{22}$ & -43 \\
\hline
\end{tabular}


Optimal phases are key information necessary for designing high-contrast substrates. It is seen from Fig.3, to achieve high contrast of $\mathrm{hBN}$, the reflectivity of substrates needs to have a phase around $-90^{\circ}$, i.e., $r=-i r_{0}$, a pure imaginary reflectivity. The phases of most conventional substrates like those shown in Fig.2a are considerably away from $-90^{\circ}\left(270^{\circ}\right)$, which is why these substrates fail to produce high visibility for hBN. With custom-designed substrates of pure imaginary reflectivity, extraordinarily high contrast can be achieved on monolayer hBN (the results will be presented in future publications).
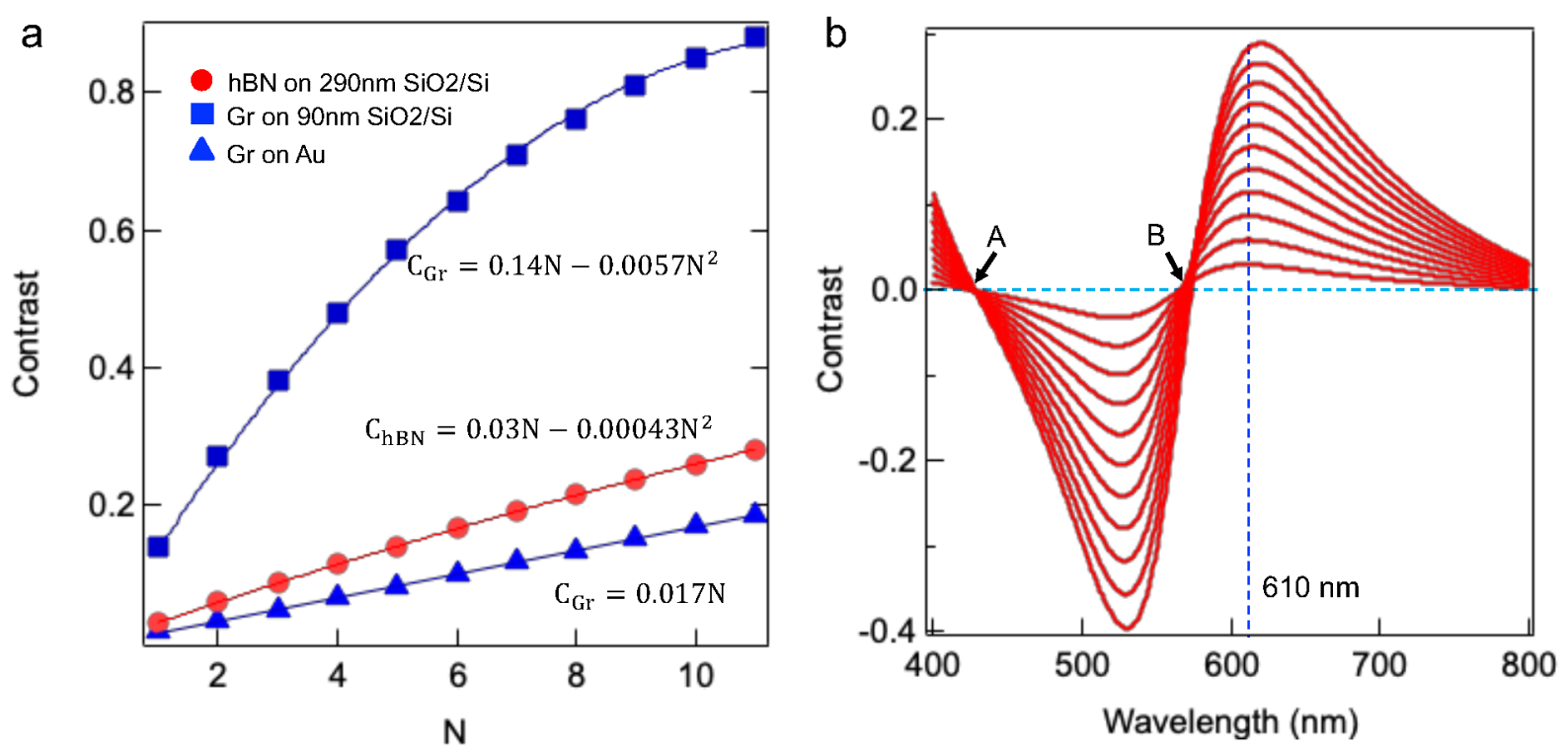

Figure 4 Contrast vs number of layers. (a) Contrast of 1-11 layer graphene films on $90 \mathrm{~nm}$ $\mathrm{SiO}_{2} / \mathrm{Si}$ (squares) showing a quadratic dependency on the number of layers, while that on $\mathrm{Au}$ substrates (triangles) is linear. $\lambda=560 \mathrm{~nm}$. (b) Contrast spectra of 1-11 layer (from low to high

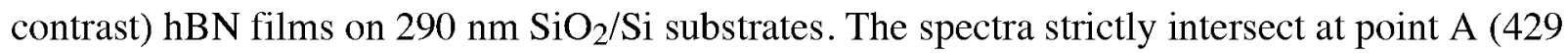
$\mathrm{nm})$ of zero contrast. Point B $(574 \mathrm{~nm})$ is a psuedo intersecting point. Contrast data extracted at 
$610 \mathrm{~nm}$ (dashed line) are presented in a (circles), showing a weak quadratic dependence on the number of layers. All the results are calculated with TE-polarization and at normal incidence.

\section{Effects of film thickness}

As shown in Eq.6a, $\chi_{0}$ is linearly proportional to $h$. Inputting Eq.6a into Eq.2, we get

$$
C=\frac{k_{0} \delta_{0} \cos \psi}{r_{0} \cos \theta} h-\left(\frac{k_{0} \delta_{0}}{2 r_{0} \cos \theta}\right)^{2} h^{2}
$$

Generally contrast is a quadratic function of $h$. In some circumstances the second term in Eq.9 could be much smaller than the first term and is negligible so that the contrast becomes linear to the thickness, as often reported in literature. ${ }^{2,3}$ This corresponds to the condition of

$$
k_{0} h \ll \frac{4 r_{0} \cos \theta|\cos \psi|}{\delta_{0}}
$$

The above condition can be matched when $r_{0}$ is not too small and $\cos \psi \neq 0$. For substrates of low reflectivity, the linear dependence is only applicable to a few layers. Figure 4a presents calculated contrasts for 1-11 layer graphene films on $90 \mathrm{~nm} \mathrm{SiO} / / \mathrm{Si}$ (squares) and Au substrates (triangles), at $\lambda=560 \mathrm{~nm}$ and normal incidence. The contrast is linear to the layer number on highreflectivity Au substrates (the condition of Eq.10 is well matched). In contrast, the dependence of contrast on thickness is quadratic on low-reflectivity $90 \mathrm{~nm} \mathrm{SiO}_{2} / \mathrm{Si}$ substrate, where linear relationship is only up to a few layers. These are consistent with Eq.9 and have been observed in experiments. ${ }^{2,3,14,23}$

As the contrast function (Eq.9) is an inverted parabolic curve, it has a maximal value,

$$
C_{\max }=\cos ^{2} \psi
$$


when

$$
h=\frac{2 r_{0} \cos \theta \cos \psi}{k_{0} \delta_{0}} \equiv h_{0}
$$

Eq.11 sets the upper limit of the maximally achievable positive contrast for a film, when the substrate and light illumination conditions are fixed. As the film thickness increases, the contrast initially increases until reaching the maximum at $h=h_{0}$, it then starts to decline after $h>h_{0}$. Cautions need to be taken when characterizing the thickness of 2D materials based on positive contrast, especially for high-visibility films such as TMDs. A low-contast image may not necessarily be a thin film. It could be a thick film beyond the optimal thickness $h_{0}$. In this case, OCS needs to be used in conjunction with other characterization techniques such as AFM or Raman for accurate results. Working with negative contrast will not have this probelem, as negative contrast is unbounded, as shown in Eq.1.

\section{Zero contrast}

Under specific conditions, contrast can be zero. According to Eq.2, this corresponds to either

$$
\chi_{0}=0
$$

or

$$
\cos \psi=\frac{\chi_{0}}{2}
$$

Based on Eq.5a, the condition of Eq.13a can only be met when $\delta=0$, which indicates $\chi=0$, regardless of film thickness $h$. This condition can be matched at a specific wavelength $\lambda_{o}$, so that $\delta\left(\lambda_{o}\right)=0$. Since it is independent of film thickness, the contrast of multilayer films will all be zero at $\lambda_{o}$, therefore the contrast spectra of multilayer films will intersect at the point of $\lambda_{o}$. Zero 
contrast could also be reached at a different wavelength when the condition of Eq.13b is matched, however, as $\chi_{0}$ is proportional to $h$, this wavelength is dependent on film thickness, therefore it is a pseudo intersecting point which can be distinguished from the true zero-contrast intersecting point. To give an example, Figure $4 \mathrm{~b}$ shows the calculated contrast spectra of 1-11 layers of hBN films on $290 \mathrm{~nm} \mathrm{SiO} 2 / \mathrm{Si}$ substrates (normal incidence, TE-polarization). All the spectra meet at the zero-contrast point A $(\lambda=429 \mathrm{~nm})$. Point $\mathrm{B}(\lambda=574 \mathrm{~nm})$ looks like a similar intersecting point, but actually is a psuedo one, as described above. The contrast data at $\lambda=610$ $\mathrm{nm}$ is extracted and plotted in Fig.4a (circles), indicating a weak quadratic dependence on the number of hBN layers. The calculated spectra well resemble those measured in experiments. ${ }^{4}$

\section{Effects of light illumination}

Optical contrast also significantly depends on the conditions of light illumination, including wavelength, polarizations and incident angles. As $\chi_{0}$ is inversely proportional to $\lambda$, contrast spectrum generally has a diminishing tail at long wavelengths, ${ }^{1-6}$ as shown in Fig.4b. For highly dispersive thin films, refractive index $n$ is strongly dependent on wavelength $\lambda$. This is often manifested as distinct features in the optical contrast spectra, as demonstrated in some TMDs. ${ }^{5,6,22}$ For stratified substrates, $r$ is sensitive to $\lambda$. As discussed above, contrast usually increases with decreasing reflectivity, therefore the maximum positive/negative contrast points in a contrast spectrum are often linked to the reflectance minima of a substrate, as shown on 90 $\mathrm{nm}$ and $290 \mathrm{~nm} \mathrm{SiO} / \mathrm{Si}$ substrates. ${ }^{1,14,23}$ The spectral position of reflectance minima can be 
shifted with varying thickness of $\mathrm{SiO}_{2}$ films, hence the contrast maxima/minima can be tuned to desirable working wavelengths.

Figure 5 demonstrates how the contrast of a monolayer graphene depends on the incident angles and polarizations. The contrasts of TE- and TM- polarizations are notably different, but both are significantly dependent on the incident angle. For TE-polarization (Fig.5a), the maximum contrast increases with incident angle (Fig.5c), whereas for TM-polarization it decreases (Fig.5d). The wavelengths of maximum contrast blueshift with incident angle for both polarizations. For TE-polarization, it shifts from $550 \mathrm{~nm}$ at $\theta=0^{\circ}$ to $408 \mathrm{~nm}$ at $\theta=74^{\circ}$, and the contrast correspondingly increases from $14.3 \%$ to $97 \%$. For TM-polarization, the wavelength of maximum contrast shifts from $550 \mathrm{~nm}$ at $\theta=0^{\circ}$ to $400 \mathrm{~nm}$ at $\theta=60^{\circ}$ (for $\theta>60^{\circ}$, the wavelength is below $400 \mathrm{~nm}$ and out of the spectral range), and accordingly the maximum contrast drops from $14.3 \%$ to $7.4 \%$.

In experiments, light is often incident from a range of angles with a spanning cone of $\theta_{0}$ defined by the NA of an objetive ( $\left.\mathrm{NA}=\sin \theta_{0}\right)$. For high numerical-aperture objectives, $\theta_{0}$ is considerbaly large, so the impacts of polarization and incident angles are pronounced, which need to be properly taken into account for accurately interpretating experimental results. ${ }^{2}$ Apart from polarization and incident angles, there is another important issue which however is often mistreated in theoretical modelling. From simple geometric optics configurations, it is obvious that light incident at different angles will have different proportions of power: those incident through the outer sector of the input aperture (corresponding to large incident angle) has higher portions of power than that incident through the center (small incident angle) of the input 
aperture..$^{2,26}$ The reflectance of light needs to be averaged with a weight-function of power with regard to the incident angle, which can be computed as, 2,26

$$
\bar{R}=\frac{2 \int_{0}^{\theta_{0}} R(\theta) \tan \theta(\sec \theta)^{2} d \theta}{\left(\tan \theta_{0}\right)^{2}}
$$

where $R(\theta)=|r(\theta)|^{2}$ is the reflectance at incident angle $\theta . \theta_{0}$ is the spanning angle defined by the NA of objective, $\mathrm{NA}=\sin \theta_{0}$. The results of Eq.14 have been demonstrated matching experimental results excellently. ${ }^{2,3}$ In literature, a variety of weight-functions of power has been used, most of them are Gaussian functions of various forms. ${ }^{4,23-25}$ The profile of Gaussian function means light incident at $\theta=0^{\circ}$ contains the maximum portion of power, which contradicts the results in Eq.14 where most light is incident at large angles and minimum at $\theta=$ $0^{\circ}$. This suggests that Gaussian functions are inappropriate weight-functions to calculate the average light reflectance measured by high-magnification objectives, which may be one of the reasons contributing to the notable discrepancy between experimental and theoretical results. ${ }^{4,23-}$ 

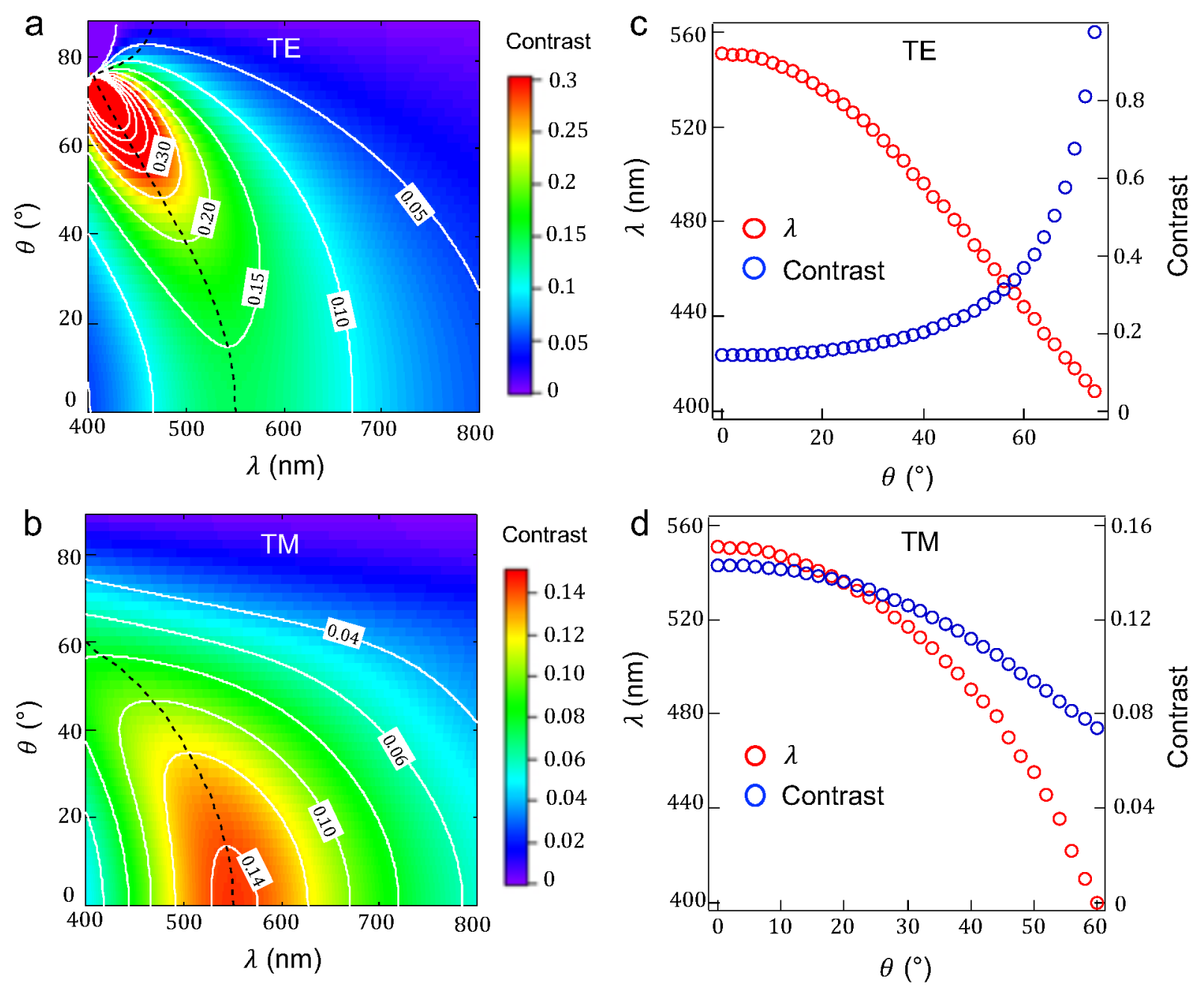

Figure 5 Effects of light illumination. (a-b) Contrast maps of a monolayer graphene on $90 \mathrm{~nm}$ $\mathrm{SiO}_{2} / \mathrm{Si}$ substrate, as functions of incident angles, wavelengths and polarizations, (c-d) The dependence of the maximum contrast and wavelength on the incident angle for TE- and TMpolarizations.

\section{CONCLUSION}

In summary, here we provide a comprehensive theoretical description of the optical contrast of atomically-thin films. The effects of substrate, thin film and light illumination conditions are fully 
investigated. It is shown that for any thin film, high contrast can be achieved on low-reflectivity substrates with optimal phases uniquely decided by the optical properties of the thin film. A universal phase map is provided, which can be used to determine the optimal phases of any given film. With the key information of optimal phases, high-contrast substrates can be designed for any thin film. It is shown that high visibility for monolayer $\mathrm{hBN}$ can be achieved on substrates with an optimal phase of $-90^{\circ}$. We also provide detailed discussions on a number of potential issues in OCS technology, such as the limitations of positive contrast, the dependence of contrast on film thickness, the zero-contrast intersecting points, and how to calculate the averaged optical reflectance measured by high-magnification objectives. The presented theoretical model is general, applicable to any planar thin films. It shows, with appropriate substrates, extraordinarily high optical contrast can be achieved on any film of any thickness.

\section{AUTHOR INFORMATION}

\section{Corresponding Author}

*Email: f.huang@qub.ac.uk

\section{ORCID}

Fumin Huang: 0000-0001-6489-9818

\section{ACKNOWLEDGMENT}

This project was supported by the UK EPSRC (grant No. EP/N025938/1).

\section{REFERENCES}

(1) Blake, P.; Hill, E. W.; Castro Neto, A. H.; Novoselov, K. S.; Jiang, D.; Yang, R.; Booth, T. J., Geim, A. K. Making graphene visible. Appl. Phys. Lett. 2007, 91, 063124.

(2) Katzen, J. M.; Velický, M.; Huang, Y.; Drakeley, S.; Hendren, W.; Bowman, R. M.; Cai, Q.; Chen, Y.; Li, L. H; Huang, F. Rigorous and accurate contrast spectroscopy for 
ultimate thickness determination of micrometer-Sszed graphene on gold and molecular sensing. ACS Appl. Mater. Interfaces. 2018, 10, 22520-22528.

(3) Velicky, M.; Hendren, W. R.; Donnelly, G. E.; Katzen, J. M.; Bowman, R. M; Huang, F. Optimizing the visibility of graphene and graphene oxide on gold with multilayer heterostructures, Nanotechnology 2018, 29, 275205.

(4) Gorbachev, R. V.; Riaz, I., Nair, R. R.; Jalil, R.; Britnell, L.; Belle, B. D.; Hill, E. W.; Novoselov, K. S.; Watanabe, K.; Taniguchi, T.; Geim, A. K., Blake, P. Hunting for monolayer boron nitride: optical and Raman signatures. Small 2011, 7, 465-468.

(5) Mao, M.; Tang, J.; Xie, L.; Wu, J.; Han, B.; Lin, J.; Deng, S.; Ji, W.; Xu, H.; Liu K.; Tong, L.; Zhang, J. Optical anisotropy of black phosphorous in the visible regime. $J$. Am. Chem. Soc. 2015, 138, 300-305.

(6) Benameur, M. M.; Radisavljevic, B.; Heron, J. S.; Sahoo, S.; Berger, H.; Kis, A. Visibility of dichalcogenide nanolayers. Nanotechnology 2011, 22, 125706.

(7) Velický, M.; Donnelly, G. E.; W. R.; McFarland, S.; Scullion, D.; DeBenedetti, W. J. I.; Correa, G. C.; Han, Y.; Wain, A. J.; Hines, M. A.; Muller, D. A.; Novoselov, K. S.; Abruña, H. D.; Bowman, R. M.; Santos, E. J. G and Huang, F. Mechanism of goldassisted exfoliation of centimeter-sized transition-metal dichalcogenide monolayers. ACS Nano 2018, 12, 10463-10472.

(8) Huang, Y.; Sutter, E.; Shi, N. N.; Zheng, J.; Yang, T.; Englund, D.; Gao, H-J; Sutter, P. Reliable exfoliation of large-area high-quality flakes of graphene and other twodimensional materials. ACS Nano 2015, 11, 10612-10620.

(9) Coleman, J. N.; Lotya, M.; O'Neill, A.; Bergin, S. D.; King, P. J.; Khan, U.; Young, K.; Gaucher, A.; De, S.; Smith, R. J. et al., Two-dimensional nanosheets produced by liquid exfoliation of layered materials. Science 2011, 331, 568-571.

(10) Novoselov, K. S.; Fal'ko, V. I.; Colombo, L.; Gellert, P. R.; Schwab, M. G.; Kim, K. A roadmap for graphene. Nature 2012, 490, 192-200.

(11) Xia, F.; Wang, H.; Xiao, D.; Dubey, M.; Ramasubramaniam, A. Two-dimensional material nanophotonics. Nat. Photon. 2010, 8, 899-907.

(12) Novosolev, K. S.; Mishchenko, A.; Carvalho, A.; Castro Neto, A. H. 2D materials and van der Waals heterostructures. Science 2016, 353, aac9439.

(13) Wang, Q. H.; Kalantar-Zadeh, K.; Kis, A.; Coleman, J. N.; Strano, M. S. Electronics and optoelectronics of two-dimensional transition metal dichalcogenides. Nat. Nano. 2012, 7, 699-712.

(14) Ni, Z. H.; Wang, H. M.; Kasim, J.; Fan, H. M.; Yu, T.; Wu, Y. H.; Feng, Y. P.; Shen, $Z$. X. Graphene thickness determination using reflection and contrast spectroscopy. Nano Lett. 2007, 7, 2758-2763.

(15) Kim, D. H.; Kim, S-J; Yu, J-S; Kim, J-H. Measuring the thickness of flakes of hexagonal boron nitride using the change in zero-contrast wavelength of optical contrast. J. Opt. Soc. Kor. 2015, 19, 503-507.

(16) Du, X. Z.; Uddin, M. R.; Li, J.; Lin, J. Y.; Jiang, H. X. Layer number dependent optical properties of multilayer hexagonal BN epilayers. Appl. Phys. Lett. 2017, 110, 092102.

(17) Pakdel, A.; Bando, Y.; Golberg, D. Nano boron nitride flatland. Chem. Soc. Rev. 2014, 43, 934-959.

(18) Yin, J.; Li, J.; Hang, Y.; Yu, J.; Tai, G.; Li, X.; Zhang, Z.; Guo, W. Boron nitride nanostructures: fabrication, functionalization and applications. Small 2016, 12, 29422968. 
(19) Giovannetti, G.; Khomyakov, P. A.; Brocks, G.; Karpan, V. M.; van den Brink, J.; Kelly, P. J. Doping graphene with metal contacts. Phys. Rev. lett. 2008, 101, 026803.

(20) Levy, N.; Burke, S. A.; Meaker, K. L.; Panlasigui, M.; Zettl, A.; Guinea, F.; Castro Neto, A. H.; Crommie, M. F. Strain-induced pseudo-magnetic fields greater than 300 Tesla in graphene nanobubbles. Science 2010, 329, 544-547.

(21) Wang, X. and Lan, S. Optical properties of black phosphorus. Adv. Opt. Photon. 2016, 8, 618-655.

(22) Li, Y.; Chemikov, A.; Zhang, X.; Rigosi, A.; Hill, H. M.; van der Zande, A. M.; Chenet, D. A.; Shih, E-M; Hone, J.; Heinz, T. Measurement of the optical dielectric function of monolayer transition-metal dichalcogenides: $\mathrm{MoS}_{2}, \mathrm{MoSe}_{2}, \mathrm{WS}_{2}$ and WSe 2. Phys. Rev. B 2014, 90, 205422.

(23) Casiraghi, C.; Hartschuh, A.; Lidorikis, E.; Qian, H.; Harutyunyan, H.; Gokus, T.; Novoselov, K. S.; Ferrari, A. C. Rayleigh imaging of graphene and graphene layers. Nano Lett. 2007, 7, 2711-2717.

(24) Bruna, M. and Borini, S. Assessment of graphene quality by quantitative optical contrast analysis. J. Phys. D: Appl. Phys. 2009, 42, 175307.

(25) Bing, D.; Wang, Y.; Bai, J.; Du, R.; Wu, G; Liu, L. Optical contrast for identifying the thickness of two-dimensional materials. Opt. Commun. 2018, 406, 128-138.

(26) Richards, B. and Wolf, E. Electromagnetic diffraction in optical systems II: structure of the image in an aplanatic system. Proc. R. Soc. London. A 1959, 253, 358-379. 


\section{TOC Graphic}

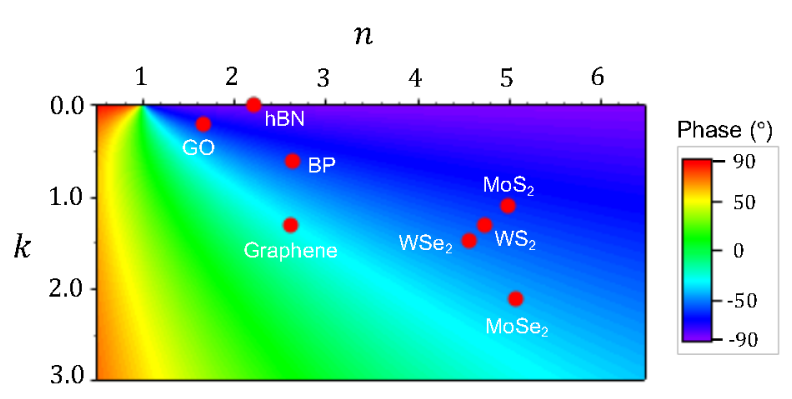

